\title{
Patient engagement and shared decision-making
}

\section{What do they look like in neurology practice?}

\author{
Melissa J. Armstrong, MD, MSc; Lisa M. Shulman, MD; Joseph Vandigo, MBA; C. Daniel Mullins, PhD
}

\begin{abstract}
Neurology is inherently patient-centered given that clinical uncertainty is common, forcing physicians, patients, and families to partner on treatment approach. An increasing emphasis on patientcentered care, patient engagement, and shared decision-making (SDM) in health care provides neurologists with an opportunity to reassess current clinical approaches to decision-making. Such assessment is not simply theoretical but has clear practice implications, with patients indicating a desire for SDM and calls for reimbursement to be tied to demonstration of SDM in practice. We present a framework for how neurologists enhance patient-centered practice by (1) eliciting patients' values and goals, (2) targeting discussion of clinical options to those values and goals, and (3) partnering with patients to make individualized decisions. We also highlight resources that facilitate SDM and examples of SDM in neurology clinical practice. Neurol Clin Pract 2016;6:190-197
\end{abstract}
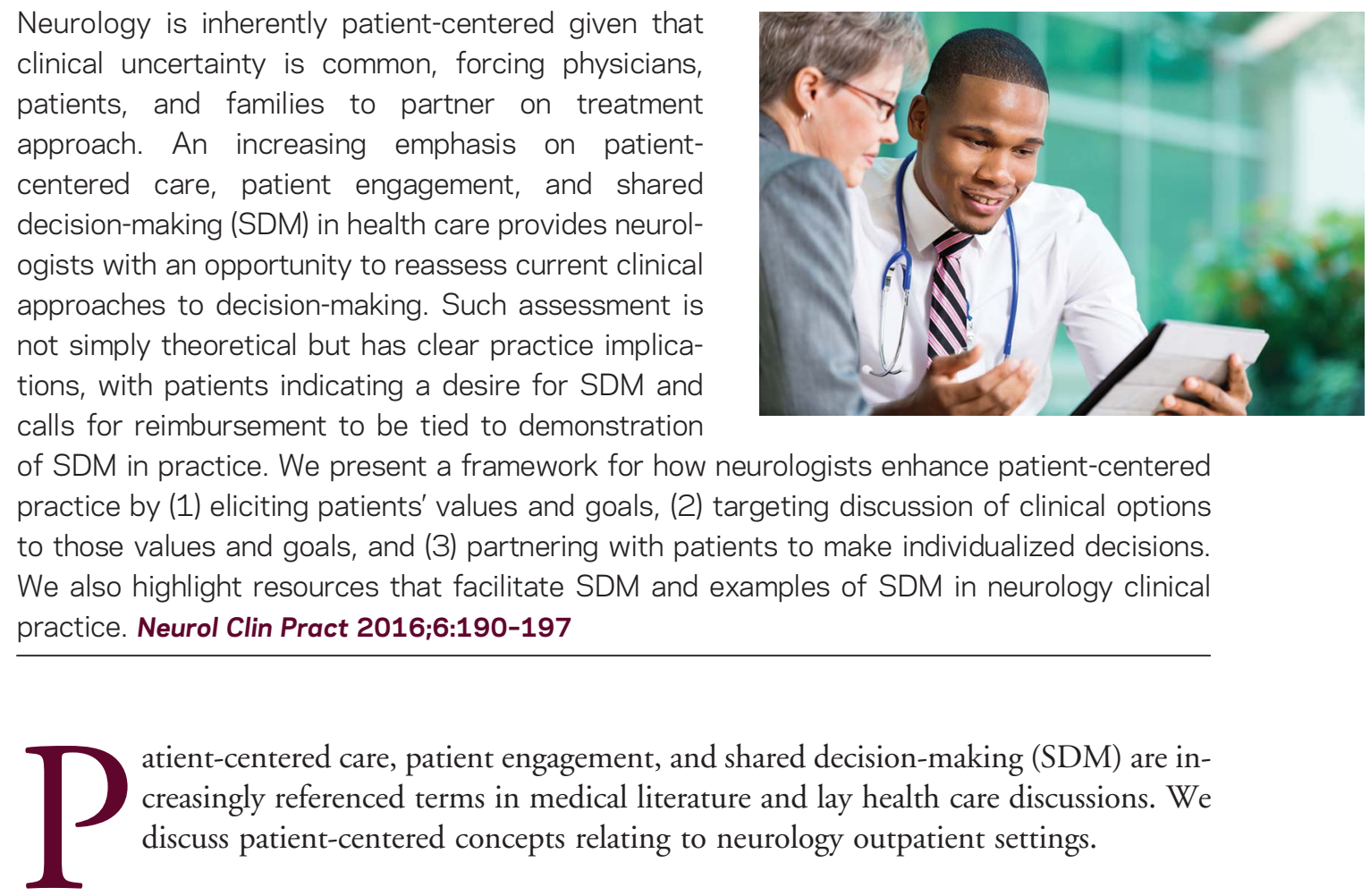

atient-centered care, patient engagement, and shared decision-making (SDM) are increasingly referenced terms in medical literature and lay health care discussions. We discuss patient-centered concepts relating to neurology outpatient settings.

The 2001 Institute of Medicine report "Crossing the Quality Chasm" is most commonly cited as bringing SDM to the forefront in American medicine. It highlighted patientcenteredness as one of 6 aims for improving high-quality health care delivery, where patient-centered care is respectful and responsive to individual preferences, needs, and values and patient values guide clinical decisions. ${ }^{1} \mathrm{SDM}$ is now described as "the pinnacle of patientcentered care." 2 SDM reflects collaboration between patients and physicians, which considers patients' values and preferences alongside evidence to make the best decisions. Patient engagement describes meaningful patient involvement in different aspects of care including research, guideline development, and clinical encounters.

Department of Neurology (MJA), University of Florida College of Medicine, Gainesville; Department of Neurology (LMS), University of Maryland School of Medicine, Baltimore; and Pharmaceutical Health Services Research Department (JV, CDM), University of Maryland School of Pharmacy, Baltimore.

Funding information and disclosures are provided at the end of the article. Full disclosure form information provided by the authors is available with the full text of this article at Neurology.org/cp.

Correspondence to: Melissa.Armstrong@neurology.ufl.edu 


\section{Patient engagement describes meaningful patient involvement in different aspects of care including research, guideline development, and clinical encounters.}

While research to date fails to demonstrate a clear effect of SDM on health outcomes, ${ }^{3}$ the foundations underpinning patient-centered care and SDM are more ethical ones, emphasizing the principles of autonomy and self-determination and the importance of patients achieving these ideals with physician assistance. Autonomy and self-determination have particular weight in neurology, where clinical uncertainty is common. There is rarely a right answer for neurologic decisions, emphasizing the importance of patients choosing the best option based on individual values and goals. For example, patients with multiple sclerosis and their physicians now have treatment options with considerations to weigh beyond efficacy, e.g., mechanism of administration, cost, and side effect profile. The value that individuals place on these considerations will differ.

Beyond the ethical mandate, there are practical reasons to perform SDM. Research suggests that most patients want to actively participate in decision-making. ${ }^{4,5}$ SDM is also highlighted in the Affordable Care Act, which mandates programs to develop, evaluate, and disseminate decision aids (DAs) that enhance SDM. Some propose that the Centers for Medicare and Medicaid Services certify and implement DAs and tie reimbursement to use of these DAs and SDM documentation. ${ }^{6}$

DAs are tools that (1) state the decision, (2) provide evidence about potential benefits, harms, and probabilities associated with different options, and (3) guide individuals through recognizing values that inform the decision. ${ }^{7}$ DAs are not stand-alone patient tools, but rather provide a framework for physicians to guide patients through decisionmaking. A Cochrane review found that DAs increased knowledge, lowered patients' decisional conflict stemming from feeling uniformed, reduced patient passivity in decision-making and the number of patients unable to decide, and resulted in more decisions for less aggressive care. ${ }^{7}$ General studies of patient-centered care and SDM show positive benefits on knowledge, satisfaction, and self-management with mixed results for clinical outcomes. ${ }^{3,8}$

\section{Patient engagement in neurologic clinical practice}

In our model for patient engagement in clinical visits (figure), each participant has a role in addressing the steps of SDM.

Role of the physician The framework includes 3 steps requiring physician action, similar to other models. ${ }^{9}$ Step 1: Physicians solicit patients' values and goals. While SDM literature often focuses on the interplay between physicians and patients addressing pros and cons of health care decisions, SDM is likely best achieved when physicians know a patient's values and goals and frame decisions in that context (table 1). Step 2: Physicians present the evidence behind health care decisions as it relates to the patient's values/goals. Physicians help patients understand how an intervention may help them achieve their goals (e.g., through symptom treatment) or how it might put goals at risk (e.g., through side effects). Step 3: Patients and physicians reach a final decision based on discussions of evidence alongside patients' values/goals.

Role of the patient Patients provide personal values and goals, informing the evidencebased discussions (step 1). They listen to options and related pros and cons presented by physicians (step 2). Ultimately, patients make the best decision for their personal situation 


\section{Figure Framework for patient engagement in clinical encounters}

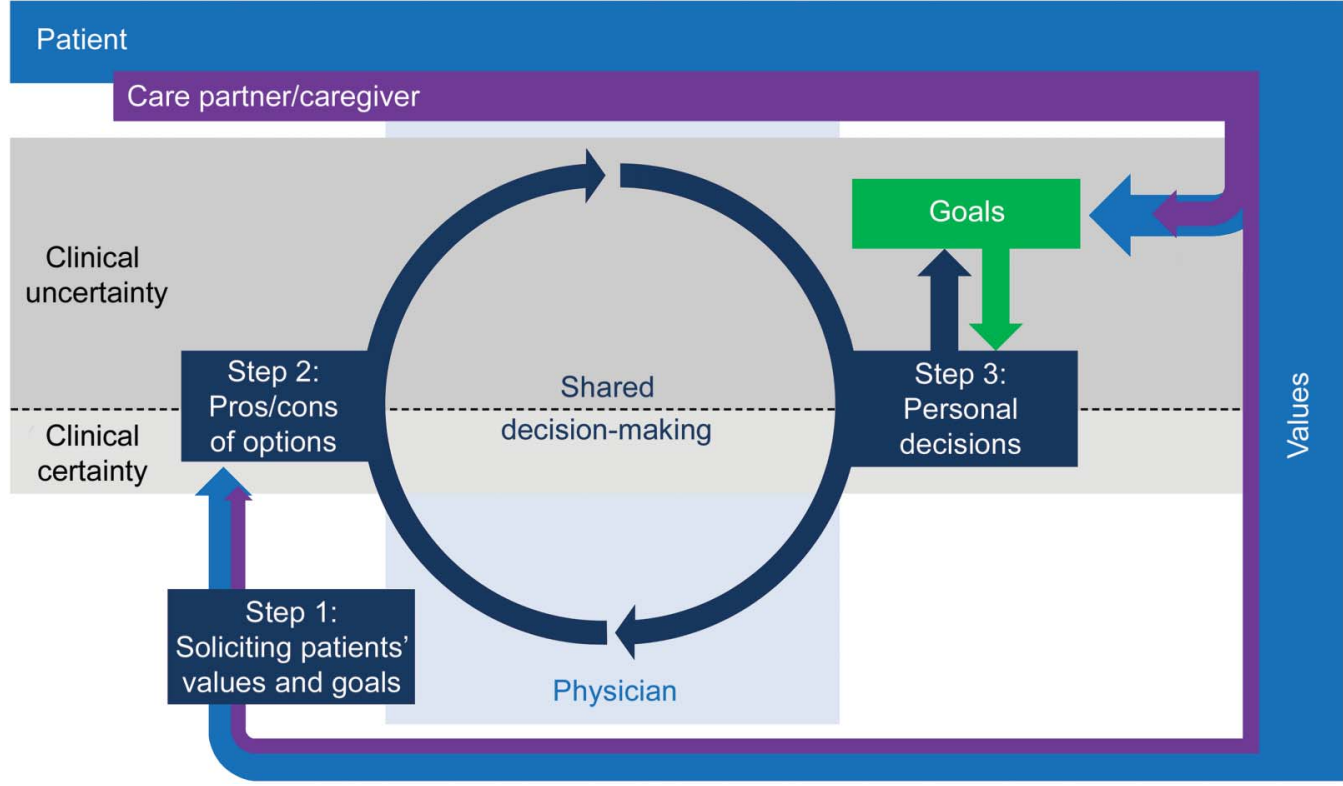

This framework for patient engagement and shared decision-making (SDM) in clinical visits shows patients at the top providing overall guidance, supported by carepartners and caregivers when relevant. Patients' values (blue) inform their goals (green) and both inform evidence-based discussions of health care options (e.g., tests, treatments) and decisions regarding the best options to achieve their goals. The 3 steps for patient engagement and SDM are outlined (boxes), with each step requiring participation by both physicians and patients. In neurology, this process most commonly occurs in the setting of clinical uncertainty, but it is relevant in situations with clinical certainty as well.

(step 3). While not all patients desire to fully engage at each step-discussed further below-most patients desire SDM.,5

Role of the carepartner/caregiver Both carepartners-family members or friends partnering in care based on existing relationship (e.g., marriage) without implying a need for assistance - and caregivers - individuals who provide assistance because of a patient's physical or cognitive limitations - have SDM roles. Patients and carepartners may share values and goals (e.g., providing for children, retirement planning) in addition to more patient-specific goals (e.g., improvement in pain) (step 1). Carepartners often have established roles in helping patients weigh pros and cons (step 2) and make major decisions (step 3). Research suggests that individuals with lower education levels may particularly rely on carepartners to assist with SDM. ${ }^{10}$ In caregiving, particularly in the context of advanced cognitive impairment, caregivers are the patient's voice. They identify the patient's previously stated goals (step 1), weigh evidence on the patient's behalf (step 2), and suggest what the patient would want if able to decide (step 3).

When patients want physicians to decide Even in studies where patients desire SDM participation, some prefer physicians to make final decisions. ${ }^{5}$ Rather than absolving physicians of SDM, however, patient reliance on physician decisions increases the need for solicitation of patients' values and goals. In this way, physicians still successfully engage patients in decision-making. If an older patient wants to remain at home but declines to make a specific clinical decision, the physician might say, "Today we discussed new medicines that could help your symptoms, but likely only a small amount. It sounds like you want keep living alone without help from your children. It might be easier for you to manage on your own if we don't add another medicine. How do you feel about that?" Here the physician engages the patient through eliciting his or her values/goals (independence, living alone 
Table 1 Examples of how solicitation of values and shared decision-making (SDM) result in different decisions in the same clinical scenario

The decision of which dopaminergic medication to start in a patient with Parkinson disease (PD) requiring symptomatic treatment is complex. Among other factors to weigh, potential advantages of starting levodopa/carbidopa include a greater effect on disability and less immediate side effects. Disadvantages include the eventual onset of motor fluctuations and dyskinesias. Benefits of starting with a dopamine agonist can include its longer duration of action (with once/day and 3 times/day formulas) and a possible delay in the onset of motor fluctuations and dyskinesias. Disadvantages include less effect on disability and side effects such as daytime somnolence and impulse control disorders. Here we present 3 examples of the same clinical scenario-an employed patient with PD initiating dopaminergic medication-to demonstrate how different values and SDM result in different decisions.

Case 1

\section{Step 1: Solicitation of patients' values/goals}

Step 2: Discussion of medical evidence for pros and cons of different decisions as informed by patients' values and goals

Step 3: Making the decision

\section{Case 2}

\section{Step 1: Solicitation of patients' values/goals}

Step 2: Discussion of medical evidence for pros and cons of different decisions as informed by patients' values and goals

Step 3: Making the decision

\section{Case 3}

\section{Step 1: Solicitation of patients' values/goals}

Step 2: Discussion of medical evidence for pros and cons of different decisions as informed by patients' values and goals

\section{Step 3: Making the decision}

Re-evaluation (repeat of steps 1-3 based on new assessment of values/goals)
A 55-year-old computer programmer with a 3-year history of tremor and bradykinesia worse on her dominant side has noticed that slowed typing and mouse use has affected her ability to successfully meet work targets. Her husband is at the appointment with her and they are concerned because he has recently lost his job and their children are nearing college age.

Her goal is to work as long as possible to support her family.

The medical information in the introduction above is discussed, with particular focus on the medications' variable effect on disability.

The patient and her husband discuss the pros and cons with the physician. They decide that it makes the most sense for her to start levodopa/carbidopa given its greater effect on disability, hoping that it will improve her typing and mouse skills enough for her to remain employed. The physician also discusses the benefit of the patient and her husband meeting with a financial planner to prepare for different scenarios relating to the husband's employment and the patient's ability to work so that they can plan for college tuition and retirement goals.

A 57-year-old salesman feels increasingly slow. He has not told his employer about his diagnosis and is uncertain when he wants to reveal that he has PD.

His goal is to keep his diagnosis private at the present time. He is extremely fearful of developing dyskinesias because he feels this would negatively affect his sales.

The medical information in the introduction above is discussed, with particular focus on the dosing schedules and side effects associated with different medications.

He chooses to start a once/day form of a dopamine agonist as this allows him to take medication before work and may be associated with a lower risk of dyskinesias in the short term.

A 65-year-old physician with a 2-year history of PD has noticed increased tremor and slowness.

He feels he needs symptomatic treatment to allow him to continue to practice. However, he is also very concerned about dyskinesias. He plans on retiring in the next year or two, but has not yet made firm plans for this.

The medical information in the introduction above is discussed, with particular discussion about medication effect on disability and recent evidence suggesting dyskinesia onset relates more to PD duration and total levodopa dose than timing of levodopa initiation.

He decides to start with a dopamine agonist as he is worried about dyskinesias even after discussion of the medical evidence.

He notices that he is drowsy every day after his lunch dose and this causes embarrassment in afternoon meetings. He decides to stop the agonist and remain off medicine for now as he plans on retiring soon and feels he can remain untreated until side effects will cause fewer issues.

These cases represent 3 employed patients with PD, each facing the same decision relating to initiation of treatment for PD symptoms. It is only by considering each patient's values (e.g., treating bradykinesia, minimizing medication doses, avoiding dyskinesias, avoiding daytime somnolence) and goals (e.g., working as long as possible, handling symptoms until upcoming retirement) that individualized treatment decisions can be made. By understanding each patient's values and goals, the neurologist can explain how the different treatment options may contribute to those values and goals or how side effects could put them at risk. 


\section{With direct-to-consumer advertising and}

\section{patient Internet research, physicians face} requests for tests or treatments in situations where there may be near-certainty that the request is not an appropriate approach.

without assistance) and targets the decision to those values/goals even when the patient declines to make the decision.

SDM in the face of clinical certainty With direct-to-consumer advertising and patient Internet research, physicians face requests for tests or treatments in situations where there may be near-certainty that the request is not an appropriate approach (e.g., situations targeted by Choosing $\left.W_{i s e l y}{ }^{11}\right)$. Physicians can use SDM to help patients understand why the requested approach does not fit the circumstance and how it will not further stated goals. In some cases, patients' preferences may be at odds with the evidence. SDM is not an easy answer in these challenging situations, but it provides an opportunity for strategies to correct misinformation. ${ }^{12}$

\section{Are neurologists practicing SDM?}

Many physicians perform SDM without calling it by name. A study of rural physicians found that $65 \%$ were unfamiliar with the term SDM, but $97 \%$ described using that approach. ${ }^{13}$ Conversely, one survey reported that $40 \%-50 \%$ of patient respondents indicated a desire for increased involvement in treatment decisions, ${ }^{14}$ suggesting that physicians may not be doing as well as they think. This likely reflects the fact that SDM performance is not a "yes" or "no" consideration, but a spectrum where physicians follow the framework to varying degrees.

\section{Resources to facilitate SDM}

Barriers to SDM include lack of familiarity and experience with SDM, physician and patient attitudes towards SDM, physician knowledge regarding evidence, lack of resources, and time. ${ }^{15}$ Health literacy - the skill needed to access, understand, and use information to promote one's health—can also impact SDM, ${ }^{16}$ with many individuals lacking understanding of probabilities commonly cited in health care decisions. ${ }^{17} \mathrm{SDM}$ tools can address some of these barriers such as lack of knowledge, training, resources, time, and health literacy. Lack of time is a commonly described barrier, ${ }^{18}$ though the Cochrane review found DA use increased consultation length by a median of only 2.55 minutes $^{7}$ and other research showed no increased appointment time from SDM. ${ }^{18}$

Option Grids ${ }^{19}$ allow physicians to develop personalized SDM outlines for decisions commonly encountered in their own practice. Prefilled Option Grids exist for some neurologic decisions, e.g., regarding surgery for hippocampal sclerosis in temporal lobe epilepsy. ${ }^{20} \mathrm{Clin}$ ical practice guidelines help physicians describe the evidence for different decisions (table 2). Each American Academy of Neurology (AAN) guideline is accompanied by a patient/family summary that can be shared within SDM, though these are not formal DAs. The AAN also has a variety of downloadable SDM tools (www.aan.com/practice/patient-engagement/). The 74 neurology-related Choosing Wisely recommendations can help neurologists explain when a test or treatment is unlikely to help. ${ }^{21}$ Organizations such as the Agency for Healthcare Research and Quality and Mayo Clinic's Center for Innovation develop more formal DAs with a particular emphasis on presenting infographics to represent risks, but few of these address neurologic topics. While tools help facilitate SDM, SDM can be achieved through discussion alone (e.g., table 1). 
Table 2 Example of using clinical practice guidelines to facilitate shared decision-making

Clinical practice guidelines provide a systematic review of the literature that provides neurologists with the information needed to guide patients through decisions.

Case 1

A 65-year-old man is due for routine colonoscopy screening. His cousin was recently diagnosed with colon cancer. He has a history of hypertension and hypercholesterolemia and a stroke 1 year ago attributed to intracranial large-artery atherosclerosis with mild residual left hemiparesis. His secondary stroke prevention includes aspirin $81 \mathrm{mg}$ daily and his gastroenterologist asked him to discuss the colonoscopy plans with his neurologist.

Step 1: Solicitation of patients' values/goals

Step 2: Discussion of medical evidence for pros and cons of different decisions as informed by patients' values and goals

Step 3: Making the decision

Case 2

Step 1: Solicitation of patients' values/goals

Step 2: Discussion of medical evidence for pros and cons of different decisions as informed by patients' values and goals

Step 3: Making the decision
The patient describes that he is very concerned about his own colon cancer risk given his cousin's recent diagnosis. At the same time, he lives with mild residual weakness and does not want to risk a recurrent vascular event.

The patient's neurologist reviews the 2013 American Academy of Neurology guideline ${ }^{22}$ and based on that guideline, discusses the following concepts with the patient:

1. He is at a risk for recurrent stroke given his prior intracranial largeartery atherosclerotic event

2. He is having a screening colonoscopy and may not need polypectomy (which has a higher risk of bleeding)

3. The risk difference for aspirin-associated bleeding with polypectomy is approximately $2.0 \%$ based on the guideline review

4. Bleeding with polypectomy is likely to have lower morbidity than recurrent stroke

The neurologist also provides the patient guideline summary (www.aan. com/guidelines)

The patient decides he is willing to accept an increased bleeding risk to avoid recurrent stroke. He chooses to proceed with colonoscopy and possible polypectomy while continuing aspirin $325 \mathrm{mg}$ daily.

A 70-year-old woman was diagnosed with breast cancer requiring mastectomy. She had a small-vessel distribution ischemic stroke associated with uncontrolled hypertension 5 years previously. She has no residual deficits and diligently controls her vascular risk factors.

The patient's primary concern is having her breast cancer effectively treated, though she wants to minimize associated risks.

Her neurologist reviews the guideline and notes that there is minimal literature for the risks associated with aspirin continuation during more invasive procedures. The neurologist counsels the patient and her surgical oncologist that (1) the patient likely has a relatively low risk of recurrent stroke with brief aspirin cessation given her stroke type and good risk factor control and (2) there is little research on bleeding risks with aspirin during invasive procedures. The surgical oncologist provides evidence regarding bleeding risks with mastectomy.

Together, they choose to stop the aspirin 7 days before the surgery, having weighed the need for mastectomy, relatively low recurrent stroke risk with temporary aspirin cessation given the details of her stroke history, lack of data regarding bleeding risks with aspirin continuation during mastectomy, and risks associated with bleeding should it occur. The importance of restarting the aspirin postoperatively is stressed, and a specific start date is decide upon.

\section{CONCLUSION}

There is an increasing emphasis on SDM that enables patients to make informed individualized health decisions targeting personal values and goals. Neurologists can adopt enhanced patientcentered practices by eliciting patients' values and goals, targeting discussion of evidence and options 
to those values/goals, and partnering with patients to collaboratively develop treatment plans. How SDM affects health outcomes, though, remains uncertain and research is ongoing. There is clear evidence that patients desire SDM; it is likely that calls for SDM will only increase in coming years.

\section{REFERENCES}

1. Institute of Medicine (IOM). Crossing the Quality Chasm: A New Health System for the 21st Century. Washington, DC: National Academy Press; 2001.

2. Barry MJ, Edgman-Levitan S. Shared decision making: pinnacle of patient-centered care. N Engl J Med 2012;366:780-781.

3. Shay LA, Lafata JE. Where is the evidence? A systematic review of shared decision making and patient outcomes. Med Decis Making 2015;35:114-131.

4. Chewning B, Bylund CL, Shah B, Arora NK, Gueguen JA, Makoul G. Patient preferences for shared decisions: a systematic review. Patient Educ Couns 2012;86:9-18.

5. Levinson W, Kao A, Kuby A, Thisted RA. Not all patients want to participate in decision making: a national study of public preferences. J Gen Intern Med 2005;20:531-535.

6. Oshima Lee E, Emanuel EJ. Shared decision making to improve care and reduce costs. N Engl J Med 2013;368:6-8.

7. Stacey D, Légaré F, Col NF, et al. Decision aids for people facing health treatment or screening decisions. Cochrane Database Syst Rev 2014;1:CD001431.

8. Rathert C, Wyrwich MD, Boren SA. Patient-centered care and outcomes: a systematic review of the literature. Med Care Res Rev 2013;70:351-379.

9. Elwyn G, Frosch D, Thomson R, et al. Shared decision making: a model for clinical practice. J Gen Intern Med 2012;27:1361-1367.

10. Smith SK, Dixon A, Trevena L, Nutbeam D, McCaffery KJ. Exploring patient involvement in healthcare decision making across different education and functional health literacy groups. Soc Sci Med 2009;69:1805-1812.

11. Cassel CK, Guest JA. Choosing Wisely: helping physicians and patients make smart decisions about their care. JAMA 2012;307:1801-1802.

12. Lewandowsky S, Ecker UK, Seifert CM, Schwarz N, Cook J. Misinformation and its correction: continued influence and successful debiasing. Psychol Sci Public Interest 2012;13:106-131.

13. King VJ, Davis MM, Gorman PN, Rugge JB, Fagnan LJ. Perceptions of shared decision making and decision aids among rural primary care clinicians. Med Decis Making 2012;32:636-644.

14. Coulter A. Do patients want a choice and does it work? BMJ 2010;341:c4989.

15. Légaré F, Ratté S, Gravel K, Graham ID. Barriers and facilitators to implementing shared decisionmaking in clinical practice: update of a systematic review of health professionals' perceptions. Patient Educ Couns 2008;73:526-535.

16. Edwards M, Davies M, Edwards A. What are the external influences on information exchange and shared decision-making in healthcare consultations: a meta-synthesis of the literature. Patient Educ Couns 2009; 75:37-52.

17. Gigerenzer G, Gaissmaier W, Kurz-Milcke E, Schwartz LM, Woloshin S. Helping doctors and patients make sense of health statistics. Psychol Sci Public Interest 2007;8:53-96.

18. Légaré F, Witteman HO. Shared decision making: examining key elements and barriers to adoption into routine clinical practice. Health Aff 2013;32:276-284.

19. Elwyn G, Lloyd A, Joseph-Williams N, et al. Option Grids: shared decision making made easier. Patient Educ Couns 2013;90:207-212.

20. Seal RP, Kynaston J, Elwyn G, Smith PE. Using an Option Grid in shared decision making. Pract Neurol 2014;14:54-56.

21. Callaghan BC, De Lott LB, Kerber KA, Burke JF, Skolarus LE. Neurology Choosing Wisely recommendations: 74 and growing. Neurol Clin Pract 2015;5:439-447.

22. Armstrong MJ, Gronseth G, Anderson DC, et al. Summary of evidence-based guideline: periprocedural management of antithrombotic medications in patients with ischemic cerebrovascular disease: report of the guideline Development Subcommittee of the American Academy of Neurology. Neurology 2013;80:2065-2069.

Received December 7, 2015. Accepted in final form January 12, 2016.

\section{AUTHOR CONTRIBUTIONS}

M.J.A.: design/conceptualization of manuscript, analysis/interpretation of concepts, drafting of manuscript, revision of manuscript. L.M.S.: analysis/interpretation of concepts, revision of manuscript. 
J.V.: analysis/interpretation of concepts, revision of manuscript. C.D.M.: analysis/interpretation of concepts, revision of manuscript.

\section{STUDY FUNDING}

M.J.A. is supported by an ARHQ K08 career development award (K08HS24159-01) and this manuscript was developed through that support.

\section{DISCLOSURES}

M.J. Armstrong has received honoraria from International Parkinson and Movement Disorder Society and American Academy of Neurology; serves on the Level of Evidence Editorial Board for Neurology; receives publishing royalties for Parkinson's Disease Improving Patient Care (Oxford University Press, 2014); serves as an Evidence-Based Medicine Methodology Consultant for American Academy of Neurology; and receives research support from Abbvie, Insightec, ARHQ, NIH, University of Maryland School of Medicine, Parkinson Study Group, Huntington Study Group, and CHDI Foundation. L.M. Shulman serves as Editor-in-Chief of the AAN Neurology Now Patient Book Series and on the editorial advisory board of Neurology Now; receives publishing royalties for Parkinson's Disease: A Complete Guide for Patients and Families, Johns Hopkins University Press: 2000, 2007, 2013; and receives research support from Kyowa Pharmaceuticals, Biotie, NIH, Michael J. Fox Foundation for Parkinson's Research, and The Rosalyn Newman Foundation. J. Vandigo receives research support from AHRQ. C.D. Mullins serves on scientific advisory boards and as a consultant for Bayer, Mundipharma, and Pfizer; serves on the speakers' bureau for Pfizer; and receives research support from Bayer, Novartis, Pfizer, AHRQ, NHLBI, and PCORI. Full disclosure form information provided by the authors is available with the full text of this article at Neurology.org/cp.

\section{Related articles from AAN physician and patient resources}

\section{Neurology ${ }^{\circledR}$ Clinical Practice}

Neurologists and end-of-life decision-making: The role of "protective paternalism" February 2015;5:6-8.

Neurologists as primary palliative care providers: Communication and practice approaches February 2016;6:40-48.

Ethical considerations in elective amputation after traumatic peripheral nerve injuries August 2014;4:280-286.

Autonomy and the "demanding encounter" in clinical neurology

April 2015;5:126-131.

Health literacy and medication awareness in outpatient neurology

February 2014;4:71-81.

\section{Neurology ${ }^{\circledR} \quad$ Neurology.org}

Challenges to ethics and professionalism facing the contemporary neurologist September 30, 2014;83:1285-1293.

\section{Neurology Now ${ }^{\circledR}$ - Neurologynow.com}

Patients First: As the new editor-in-chief, I intend to keep Neurology Now informative, relevant, and focused on YOU

February/March 2015;11:6. 